\title{
System-level line-edge roughness limits in extreme ultraviolet lithography
}

\author{
Patrick P. Naulleau, ${ }^{1}$ Dimitra Niakoula, ${ }^{1}$ and Guojing Zhang ${ }^{2}$ \\ ${ }^{1}$ Center for X-Ray Optics, Lawrence Berkeley National Laboratory, Berkeley, CA 94720 \\ ${ }^{2}$ Intel Corporation, Santa Clara, CA 95052
}

\begin{abstract}
As critical dimensions shrink, line edge and width roughness (LER and LWR) become of increasing concern. Traditionally LER is viewed as a resist-limited effect; however, as critical dimensions shrink and LER requirements become proportionally more stringent, system-level effects begin to play an important role. Recent advanced EUV resist testing results have demonstrated lower bounds on achievable LER at the level of approximately 2 to $3 \mathrm{~nm}$. Here we use modeling to demonstrate that a significant portion of this low bound may in fact be do to system-level effects and in particular the mask. Of concern are both LER on the mask as well as roughness of the multilayer reflector. Modeling also shows roughness (flare) in the projection optics not to be of concern.
\end{abstract}

Keyword: extreme ultraviolet, lithography, multilayer, mask, line-edge roughess 


\section{Introduction}

Line edge and width roughness (LER and LWR), has become an issue of increasing concern as projection lithography techniques push to smaller and smaller feature sizes. This poses significant challenges to the development of photoresist for next-generation lithography techniques such as 193-nm immersion and extreme-ultraviolet (EUV) lithography. For example at the 32-nm DRAM half-pitch fabrication node, the International Technology Roadmap for Semiconductors [1] calls for a LWR in resist of less than $1.7 \mathrm{~nm}$ (LER $<1.2 \mathrm{~nm}$ assuming uncorrelated edges). Historically, LWR is viewed as a resist-limited effect; however, as LWR requirements approach single-nm values, systemlevel effects may begin to play an important role. Examples of such system-level effects include LER on the mask [2], mask reflector surface roughness [3, 4], and projection optics scatter [5]. Recent advanced EUV resist testing using the SEMATECH Berkeley EUV microfield exposure tool (MET) $[6,7]$ has demonstrated a lower bound on achievable LER at the level of approximately $2 \mathrm{~nm}$. Here we present detailed modeling results evaluating the importance of the above mentioned system-level effects in the observed LER lower limit.

\section{Modeling methodology}

The SEMATECH Berkeley MET imaging characteristics are modeled using scalar aerial image computation software based on the partially-coherence image formation equations [8]. Similar capabilities can also be obtained through the use of commercial modeling packages such as Prolith [9] or Solid EUV [10]. The relatively small numerical aperture utilized by EUV systems (the MET has a numerical aperture of 0.3 ) enables us to use scalar modeling as well as the thin mask approximation. The mask LER used in the modeling is determined from a scanningelectron micrograph (SEM) of coded 50-nm equal lines-space features on a characteristic MET $5 \times$ mask (Fig. 1). The line edges are extracted from the SEM and a grayscale simulation mask is 
generated (Fig. 2). Note the tone reversal from the SEM to the generated simulation mask; lighter regions in the SEM correspond to the absorber, thus become dark in the generated simulation mask. In the simulation analysis to follow, we consider only the center 5 lines for LER analysis. Grayscale is used on the simulation mask to define the edge transition region enabling higher fidelity in the replication of the LER. In a strictly binary mask the edge position resolution is determined solely by the pixel size, whereas for grayscale edge transition we can effectively achieve sub-pixel edge positioning enabling high fidelity LER reproduction at reasonable sampling levels. The size of the final simulation mask is $1020 \times 1020$ pixels. The LER of the mask is $8.9 \mathrm{~nm}$ in mask coordinates and $1.8 \mathrm{~nm}$ in wafer coordinates.

Next we describe the modeling of the mask multilayer or phase roughness. Masks used in EUV lithography are reflective and are rendered so through the deposition of a multilayer coating typically comprised of 40 or more bilayers [11]. If one starts with knowledge of the uncoated substrate surface, multilayer growth models can be used $[12,13]$ to predict the coating properties throughout the stack. From the calculated coating properties, rigorous electromagnetic field modeling could be used to calculate the electric field reflected from the mask [14-16]. Such an approach, however, would be extremely time consuming. In most cases relevant to the moderate roughness of interest here, the effect of the rough mask can be readily modeled as a pure phase distribution, where the phase is determined from the geometric path-length differences imparted by assuming the EUV light to be reflected from the top surface of the mask [17]. In practice, this simplification works because the vast majority of the non-conformal multilayer growth occurs within a small number of layers closest to the substrate. Within the EUV penetration region of a typical 40 or more bilayer multilayer, the layer growth tends to be conformal for roughnesses of interest here. Using this simplified approach, one only needs to 
measure the topographic profile of the final multilayer-coated mask.

Figure 3 shows an atomic force micrograph (AFM) of the multilayer coated mask blank. The full rms roughness over the $2-\mu \mathrm{m}$ scan is $0.24 \mathrm{~nm}$ and the peak to valley roughness is 2.16 $\mathrm{nm}$. The rms slope error computed from the AFM is $1.3 \mathrm{mrad}$, approximately twice the proposed industry specification. Figure 4 shows the isotropic power spectral density (PSD) of the roughness and Fig. 5 shows the synthesized mask with phase depicted as grayscale. The mask phase error is synthesized from the PSD in Fig. 4 to achieve the same mask size and sampling as used for the rough line simulation mask in Fig. 2. As discussed below, for simulation purposes, we also consider a mask with ideally smooth lines and the same rough multilayer as used in Fig. 5.

Finally, optic scatter (flare) is modeled by adding phase roughness to the pupil mask. The pupil mask further includes information on the optic aberrations and obscuration. The optic wavefront quality is approximately $\lambda / 15$ and the flare within the $200 \times 600 \mu \mathrm{m}$ printed field is approximately 7\%. Details on the flare and aberrations in the SEMATECH Berkeley MET can be found in the literature [18-21].

\section{Modeling results}

In order to explicitly study the relative importance of the various mask contributors to LER, in the cases to follow we consider three different simulation masks: smooth lines rough multilayer (Mask 1), rough lines smooth multilayer (Mask 2), and rough lines rough multilayer (Mask 3). Figure 6 shows results for three different illumination settings: annular (0.35-0.55) [Fig. 6(a)], monopole $[\sigma=0.15$, offset $=(015,-0.45)]$ [Fig. $6(\mathrm{~b})]$, and dipole $[\sigma=0.2$, offset $=(0.36,0.36)]$ [Fig. 6(c)]. For the lowest coherence case (annular), we see the in-focus LER to be dominated by 
the LER on the mask whereas as we move out of focus the multilayer roughness begins to dominate. For the higher coherence settings, the multilayer roughness dominates throughout focus. In all cases the effect of the mask LER is relatively constant through focus, whereas the multilayer effect varies strongly with focus. As one would expect, the LER obtained from Mask 3 is approximately the quadrature sum of the LER obtained from Masks 1 and 2 independently. For annular illumination, the LER at best focus is approximately $1.4 \mathrm{~nm}$ whereas the higher coherence settings show LER values greater than $2 \mathrm{~nm}$.

Figure 7 shows a direct comparison of the various illumination settings with Mask 3. Two additional annular settings are also considered. Again it is clear that from the perspective of LER, the lower the coherence the better.

The final potential LER source we consider is roughness from the projection optics (or flare) as suggested in Ref. [5]. Figure 8 shows a direct comparison of the flare and no-flare cases with Mask 3 for various illumination settings (annular, monopole, and dipole). As evidenced by the modeling results, the effect of flare (projection optics scatter) is negligible. For the annular case, which is lowest coherence condition [Fig. 8(a)] the flare and no flare results are virtually indistinguishable. For the higher coherence cases of monopole and dipole, slight differences can be seen both increasing and decreasing the observed LER through focus. We note that unlike roughness on the mask, the effect of pupil roughness or scatter would be expected to be insensitive to focus in terms of magnitude.

\section{Discussion}

The results presented here have demonstrated the importance of mask effects in terms of the ultimate LER capabilities of EUV exposure tools. Depending on the illumination conditions, mask multilayer roughness and/or mask LER play important roles. In general, for lower coherence settings such as 
annular, mask LER is dominant, whereas for high coherence settings such as monopole and dipole, mask multilayer roughness is dominant. In all cases, mask multilayer roughness is dominant in terms of through focus behavior. This strong illumination dependence is due to the fact that the LER caused by mask multilayer roughness arises from coherent speckle effects. The impact is that as higher coherence low- $k_{1}$ type illumination settings are used to improve resolution, the resulting mask induced LER increases.

Another implication of the dominance of the mask multilayer roughness is that accurate determination of the true roughness is crucial, this measurement, however, can be complicated since all we can readily measure is the AFM roughness before and/or after multilayer coating. Having the pre-coating roughness we need to rely on complicated smoothing model which require knowledge of the coating characteristics which may be viewed as proprietary by the mask manufactures. Working from the post-coating roughness, as we have done here, we need to rely on the assumption that the measured surface roughness is indeed representative of the stack replicated roughness which directly impacts the phase as compared to simple capping layer roughness, for example, which would couple to phase much more weakly. We note that such issues could, in principle, be addressed using an EUV aerial imaging microscope with well known coherence properties.

As discussed in the modeling methodology section, the mask multilayer roughness is approximately a factor of 2 larger than specifications. It is instructive to consider what LER performance we might expect if the mask had met the specifications. To this end, we simply scale down the phase roughness by a factor of two and recompute the resulting aerial images and LER. Figure 9 shows the results for annular $(0.35-0.55)$, monopole $[\sigma=0.15$, offset $=(0,0.45)]$, and dipole $[\sigma=0.2$, offset $=(0.36,0.36)]$. Comparing the results to those shown in Fig. 6 , we see that as expected the annular case remains dominated by mask LER, but this time through an even wider focus range. 
Moreover, the monopole case is now also mask LER dominated. For the dipole case, however, the multilayer roughness still remains dominant. The best focus LER value for annular and monopole is approximately $1.2 \mathrm{~nm}$, whereas dipole has a best-focus LER of approximately $1.6 \mathrm{~nm}$.

Finally we consider the potential effect of the mask-induced LER on current resist characterization results. Recent EUV resist testing results have shown clear evidence of an asymptotic behavior of LER reduction as a function of dose leveling out at an LER of approximately $2 \mathrm{~nm}$ or slightly larger. Figure 10 depicts such data as collected from exposure results from the SEMATECH Berkeley MET. The vast majority of these results are obtained with annular or monopole illumination. Figure 11 shows the same plot but with the mask-induced LER term, as modeled here, removed. The mask-induced LER is assumed to add in quadrature with the remaining LER terms. In Fig. 11(a), a 1.43-nm LER component is removed, corresponding to lowest computed LER for annular illumination and in Fig. 11(b), a 1.99-nm LER term is removed corresponding to monopole. Despite the removal of significant LER magnitudes, the data shows that achieving the ITRS target of 1.2-nm LER for the 32-nm node still remains quite challenging.

In summary, present mask limitations dictate that numerical compensation for these effects is required in order to achieve accurate resist LER characterization below levels of approximately 3.5 $\mathrm{nm}$. It is important to note, however, that the mask presently used in the SEMATECH Berkeley MET has a multilayer roughness that is approximately twice the proposed 32-nm node specification and an absorber LER approximately 7.5 times larger than specification [1] (8.9 nm measured compared to $1.18 \mathrm{~nm}$ specification). Achieving the target mask specifications in terms of roughness and LER will be crucial to reaching the ultimate manufacturing goal for LER. Finally, the simulation also shows that the effect of projection-optics scatter is negligible in terms of aerial-image LER, even under highcoherence illumination. 
The authors are greatly indebted to Paul Denham, Brian Hoef, Gideon Jones, Jerrin Chiu, and Ken Goldberg for expert support with the exposure tool, and to the entire CXRO staff for enabling this research. The authors are also grateful to Kim Dean of SEMATECH for program support. This work was supported by SEMATECH and carried out at Lawrence Berkeley National Laboratory's Advanced Light Source, which is supported by the DOE, Office of Science, Basic Energy Sciences. 


\section{References}

1. International Technology Roadmap for Semiconductors, 2006 Update, (http://www.itrs.net/Links/2006Update/2006UpdateFinal.htm)

2. P. Naulleau and G. Gallatin, "The line-edge roughness transfer function and its application to determining mask effects in EUV resist characterization,” Appl. Opt. 42, 3390-3397 (2003).

3. N. Beaudry, T. Milster, "Effects of mask roughness and condenser scattering in EUVL systems,” Proc. SPIE. 3676, 653-662 (1999).

4. P. Naulleau, "The relevance of mask-roughness-induced printed line-edge roughness in recent and future EUV lithography tests," Appl. Opt. 43, 4025-4032 (2004).

5. H. Solak, Y. Yang, and F. Cerrina, "Observation of speckle patterns in extreme ultraviolet imaging,” J. Vac. Sci. \& Technol. B 19, 2406-2411 (2001).

6. P. Naulleau, K. Goldberg, E. Anderson, K. Bradley, R. Delano, P. Denham, B. Gunion, B. Harteneck, B. Hoef, H. Huang, K. Jackson, G. Jones, D. Kemp, A. Liddle, R. Oort, A. Rawlins, S. Rekawa, F. Salmassi, R. Tackaberry, C. Chung, L. Hale, D. Phillion, G. Sommargren, J. Taylor, "Status of EUV microexposure capabilities at the ALS using the 0.3-NA MET optic," Proc. SPIE 5374, 881-891 (2004).

7. P. Naulleau, C. Anderson, K. Dean, P. Denham, K. Goldberg, B. Hoef, B. La Fontaine, T. Wallow, "Recent results from the Berkeley 0.3-NA EUV microfield exposure tool," Proc SPIE 6517, 65170V (2007).

8. J. W. Goodman, Statistical Optics, John Wiley and Sons, New York, 1985, Chap. 7, 286360.

9. Prolith is a registered trademark of KLA-Tencor Corporation, 160 Rio Robles, San Jose, California 95134. 
10. Solid-CEUV is a registered trademark of SIGMA-C GmbH, Thomas-Dehler-Str. 9, 81737 München, Germany.

11. J. H Underwood and T. W. Barbee, Jr., "Layered synthetic microstructures as Bragg diffractors for X rays and extreme ultraviolet: theory and predicted performance," Appl. Opt. 20, 3027-3034 (1981).

12. D. Stearns, "Stochastic model for thin film growth and erosion," Appl. Phys. Lett. 62, 17451747 (1993).

13. D. Stearns, E. Gullikson, "Nonspecular scattering from extreme ultraviolet multilayer coatings,” Physica B 283, 84-91 (2000).

14. Z. Zhengrong, K. Lucas, J. Cobb, S. Hector, A. Strojwas, "Rigorous EUV mask simulator using 2D and 3D waveguide methods," Proc. SPIE 5037, 494-503 (2003).

15. A. Erdmann, C. Kalus, T. Schmoller, A. Wolter, "Efficient simulation of light diffraction from three-dimensional EUV masks using field decomposition techniques," Proc. SPIE 5037, 482-493 (2003).

16. T. Pistor T, Y. Deng, A. Neureuther, "Extreme ultraviolet mask defect simulation: Lowprofile defects,” J. Vac. Sci. \& Technol. B 18, 2926-2929 (2000).

17. E. Gullikson, C. Cerjan, D. Stearns, P. Mirkarimi, D. Sweeney, "Practical approach for modeling extreme ultraviolet lithography mask defects,” J. Vac. Sci. Technol. B 20, 81-86 (2002).

18. J. Cain, P. Naulleau, E. Gullikson, C. Spanos, "Lithographic characterization of the flare in the Berkeley 0.3-numerical aperture extreme ultraviolet microfield optic,” J. Vac. Sci. \& Technol. B 24, 1234-1237 (2006). 
19. K. Goldberg, P. Naulleau, P. Denham, S. Rekawa, K. Jackson, E. Anderson and J. Liddle, “At-Wavelength Alignment and Testing of the 0.3 NA MET Optic,” J. Vac. Sci. \& Technol. B 22, 2956-2961 (2004).

20. P. Naulleau, J. Cain, K. Goldberg, "Lithographic characterization of the spherical error in an EUV optic using a programmable pupil fill illuminator,” Appl. Opt. 45, 1957-1963 (2006).

21. P. Naulleau, J. Cain, K. Goldberg, "Lithographic characterization of the field dependent astigmatism and alignment stability of a 0.3 numerical aperture EUV microfield optic,” J. Vac. Sci. \& Technol. B 23, 2003-2006 (2005). 


\section{List of Figures}

Fig. 1. Source scanning electron micrograph (SEM) used for determination of mask LER used in modeling. The selected feature size is coded 50-nm equal lines-space features on a $5 \times$ mask.

Fig. 2. Generated grayscale simulation mask based on SEM in Fig. 1. Note the tone reversal from the SEM to the generated simulation mask; lighter regions in the SEM correspond to the absorber, thus become dark in the generated simulation mask.

Fig. 3. Atomic force micrograph (AFM) of the multilayer coated mask blank. The full rms roughness over the $2-\mu \mathrm{m}$ scan is $0.24 \mathrm{~nm}$ and the peak to valley roughness is $2.16 \mathrm{~nm}$. The rms slope error computed from the AFM is $1.3 \mathrm{mrad}$, approximately twice the proposed industry specification.

Fig. 4. Isotropic power spectral density (PSD) of the roughness as computed from AFM in Fig. 3.

Fig. 5. Synthesized multilayer phase roughness depicted as grayscale. The mask phase error is synthesized from the PSD in Fig. 4 to achieve the same mask size and sampling as used for the rough line simulation mask in Fig. 2.

Fig. 6. Simulation results for three different masks [smooth lines rough multilayer (Mask 1), rough lines smooth multilayer (Mask 2), and rough lines rough multilayer (Mask 3)] and three different illumination settings: annular $(0.35-0.55)(a)$, monopole $[\sigma=0.15$, offset $=(0,0.45)](b)$, and dipole $[\sigma=0.2$, offset $=(0.36,0.36)](\mathrm{c})$.

Fig. 7. Direct comparison of the various illumination settings with Mask 3. Two additional annular settings are also considered.(0.3-0.7 and 0.43-0.46).

Fig. 8. Direct comparison of the flare and no-flare cases with Mask 3 for various illumination settings [annular (a), monopole (b), and dipole (c)]. The effect of flare (projection optics scatter) is negligible. 
Fig. 9. Computed aerial image LER under various illumination conditions for Mask 3 resynthesized to meet industry specifications for mask roughness. The illumination settings are annular $(0.35-0.55)$, monopole $[\sigma=0.15$, offset $=(0,0.45)]$, and dipole $[\sigma=0.2$, offset $=(0.36,0.36)]$.

Fig. 10. Summary of EUV resist LER testing results form the SEMATECH Berkeley MET over the past few years. The vast majority of these results are obtained with annular or monopole illumination.

Fig. 11. Data from Fig. 10 with the mask-induced LER term removed. The mask-induced LER is assumed to add in quadrature with the remaining LER terms. In (a), a 1.43-nm LER component is removed, corresponding to lowest computed LER for annular illumination and in (b), a 1.99-nm LER term is removed corresponding to monopole. 
Naulleau, et al., Fig. 1

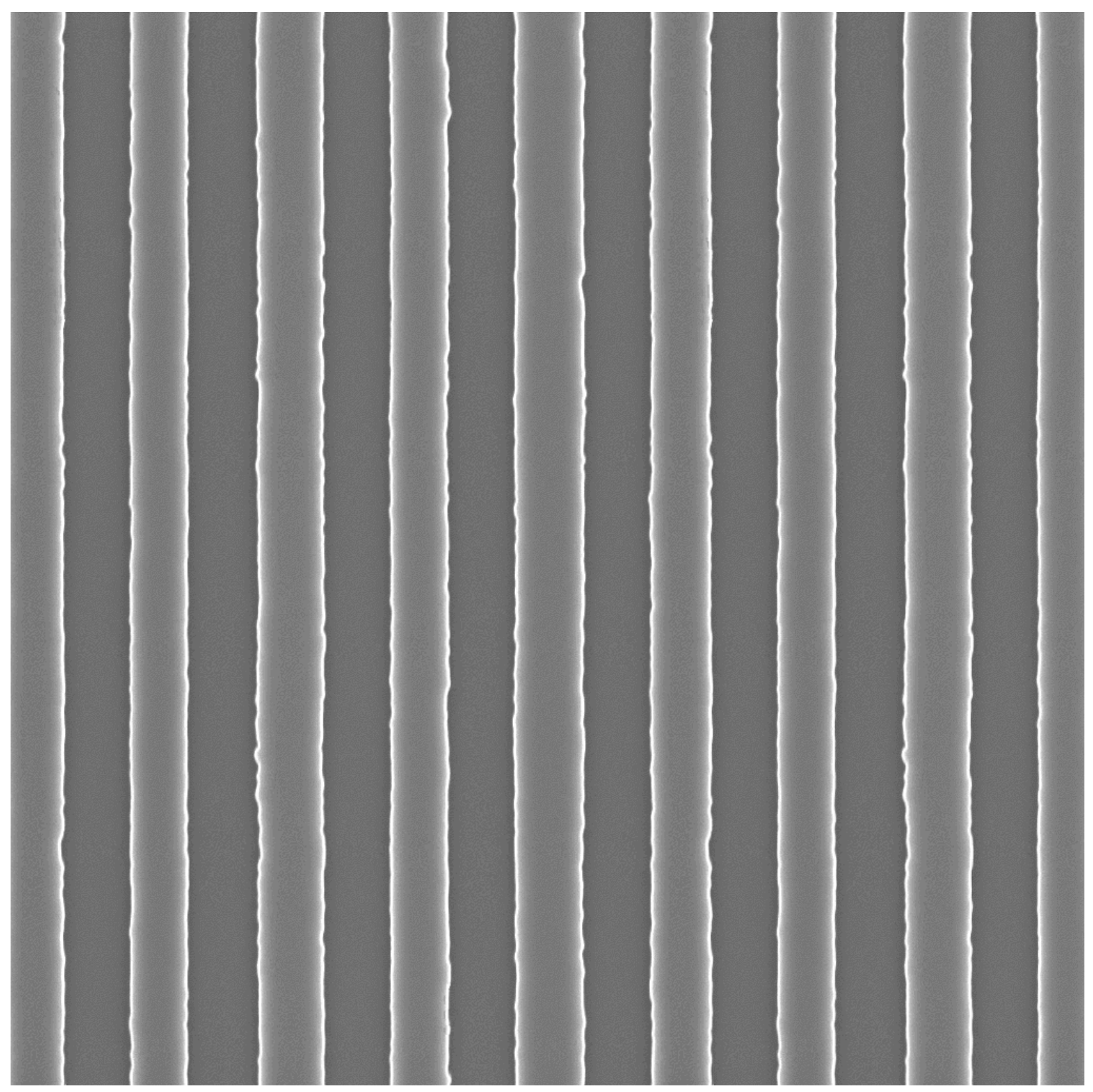


Naulleau, et al., Fig. 2
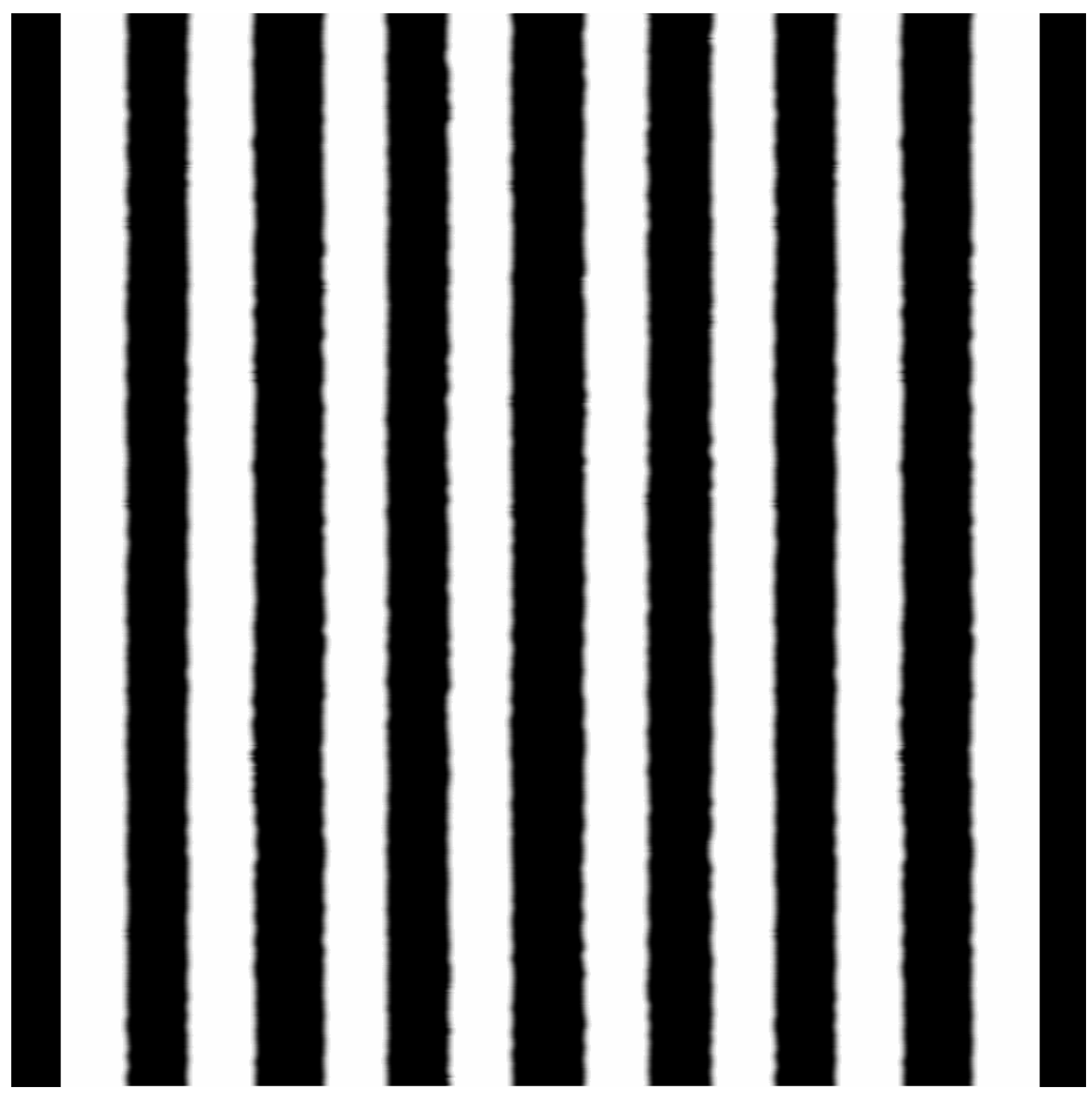
Naulleau, et al., Fig. 3

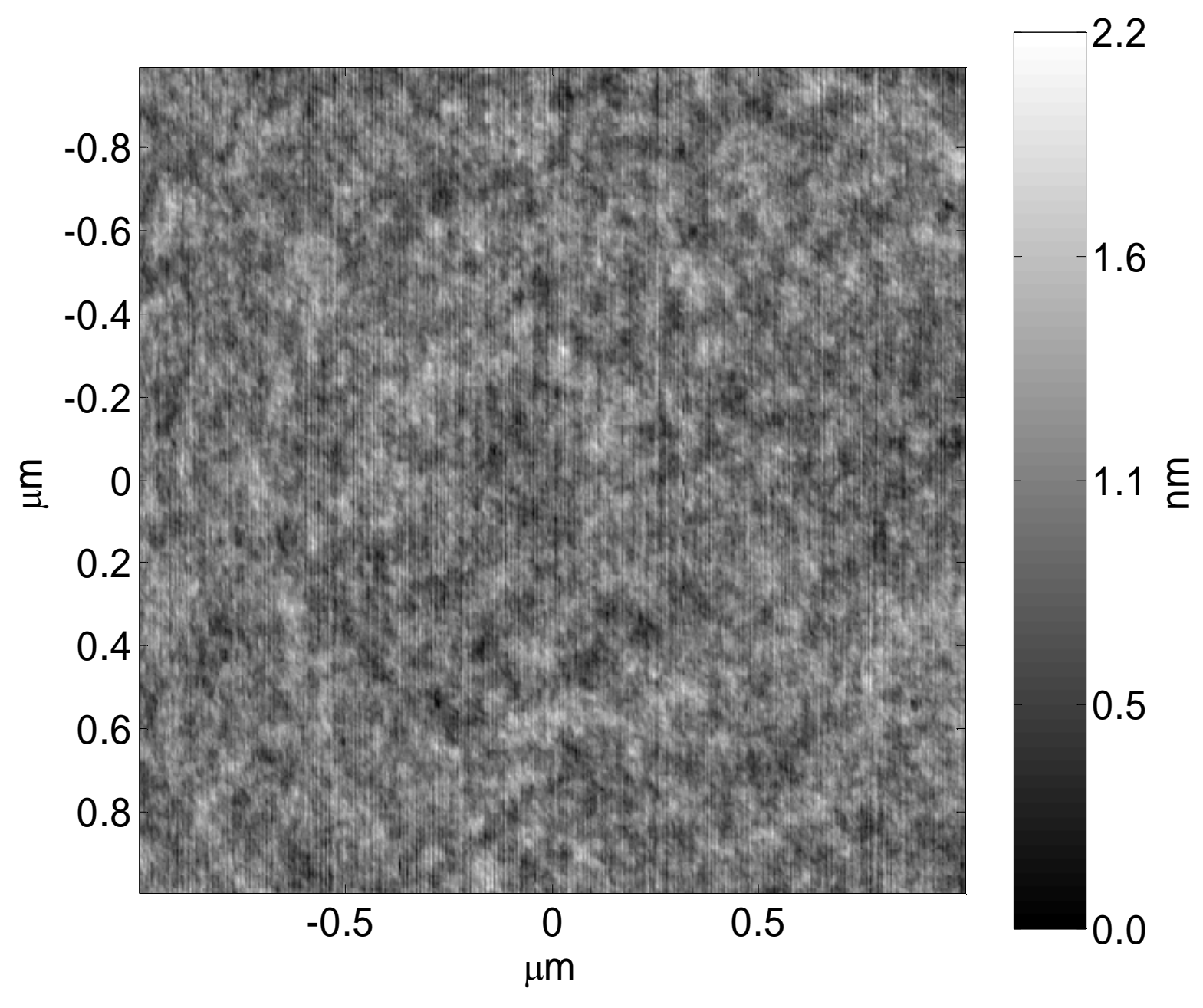


Naulleau, et al., Fig. 4

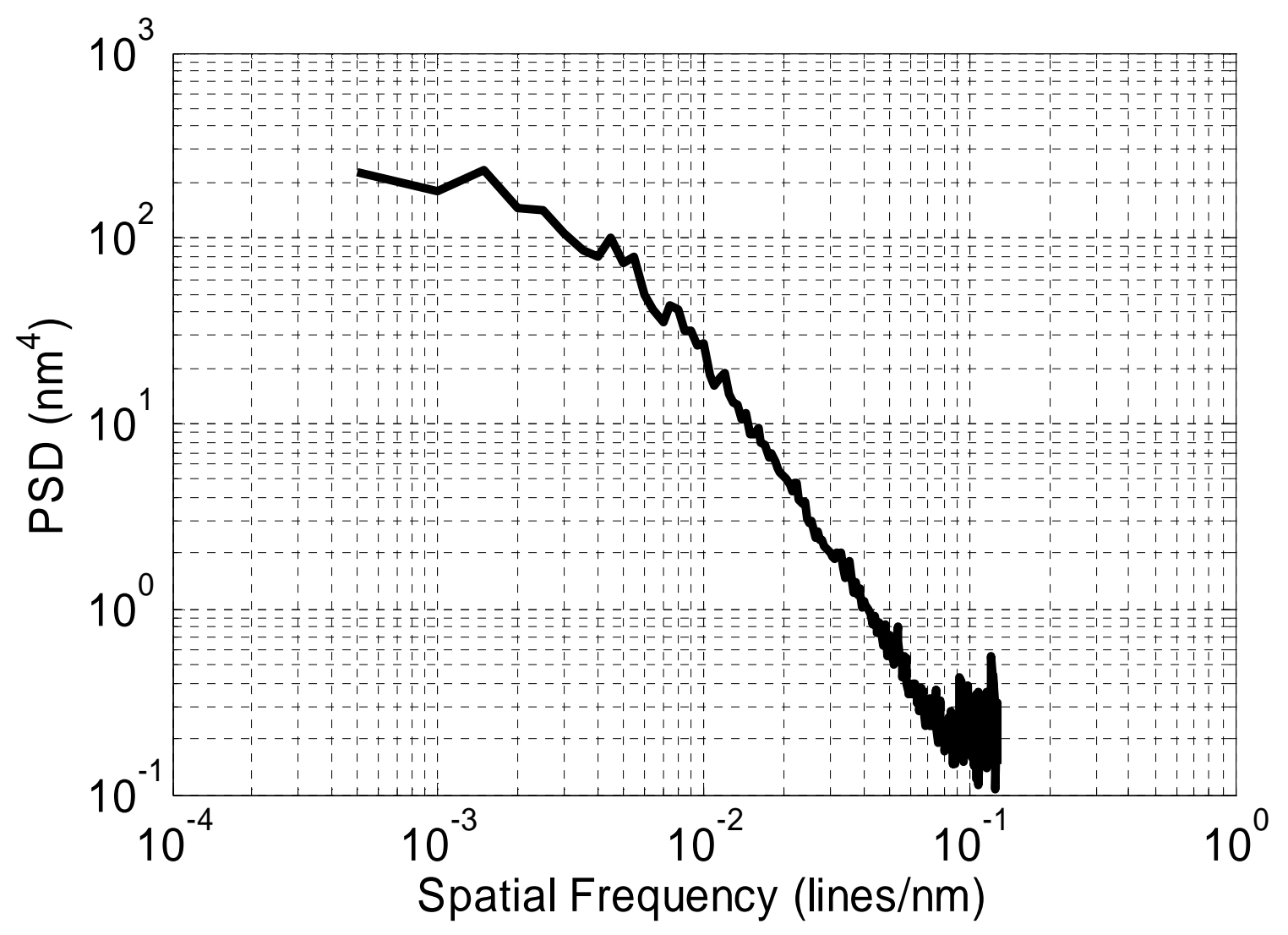


Naulleau, et al., Fig. 5

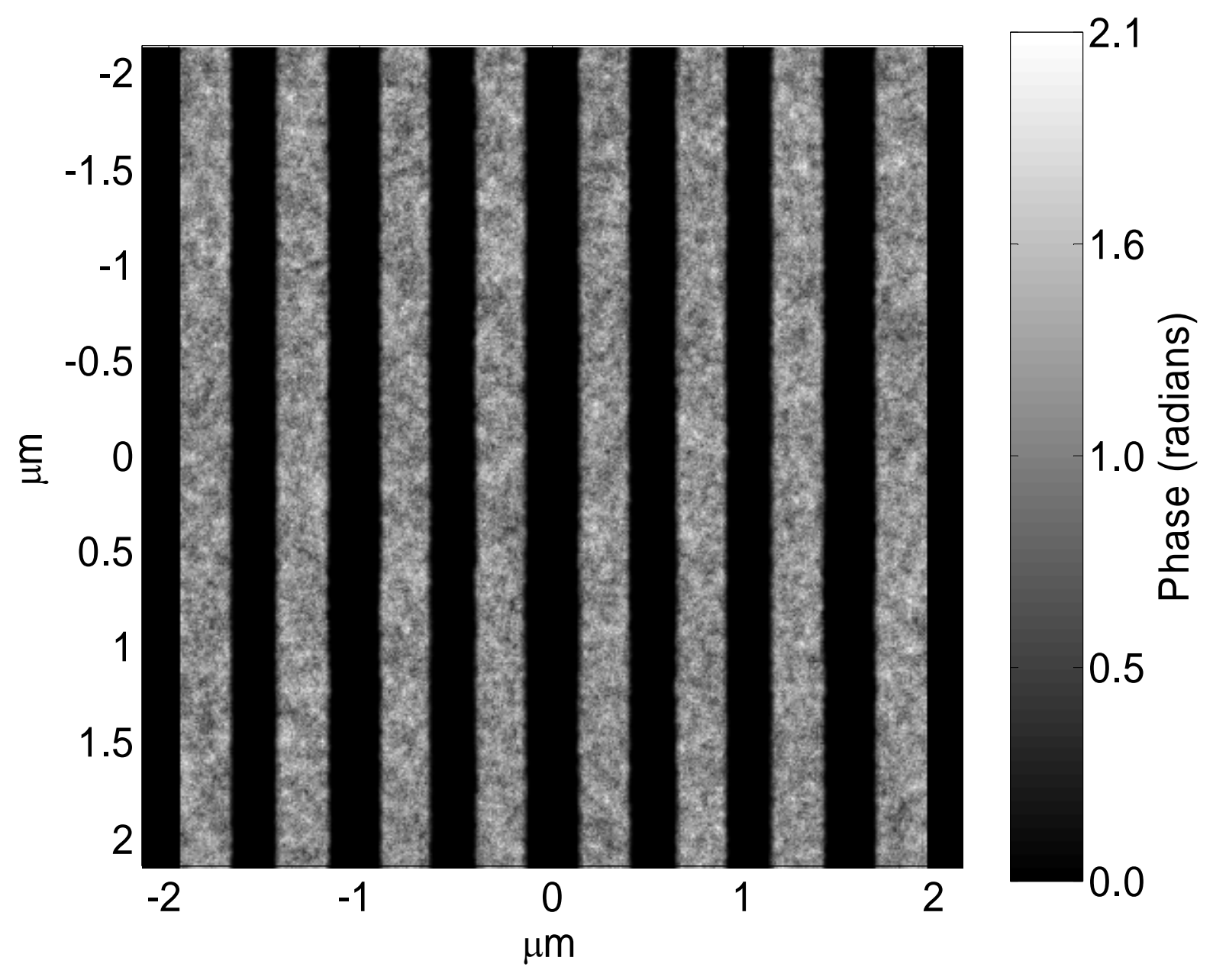




\section{Naulleau, et al., Fig. 6}

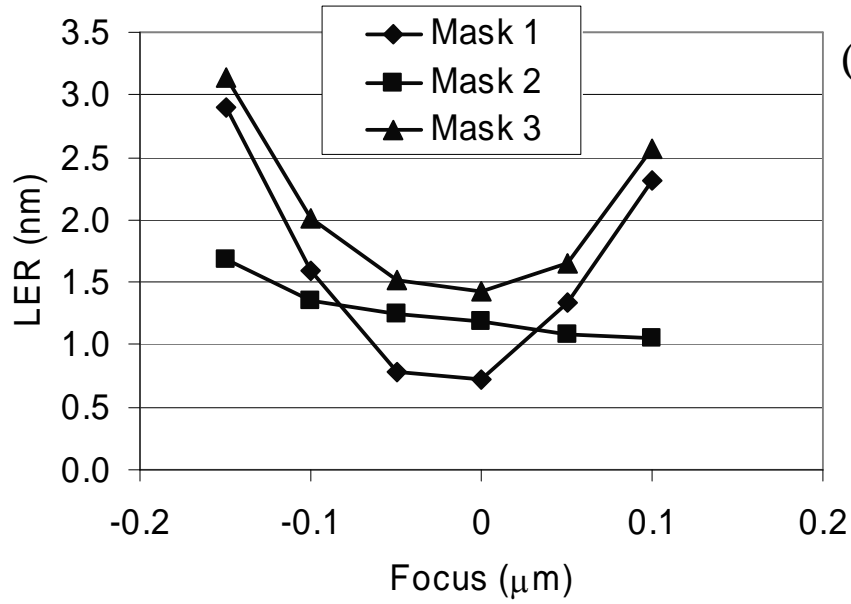

(a)

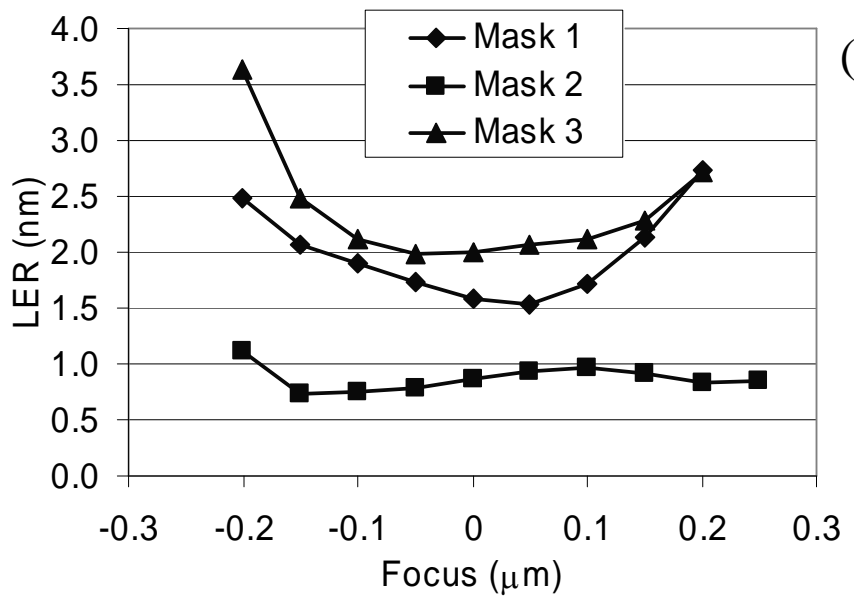

(b)

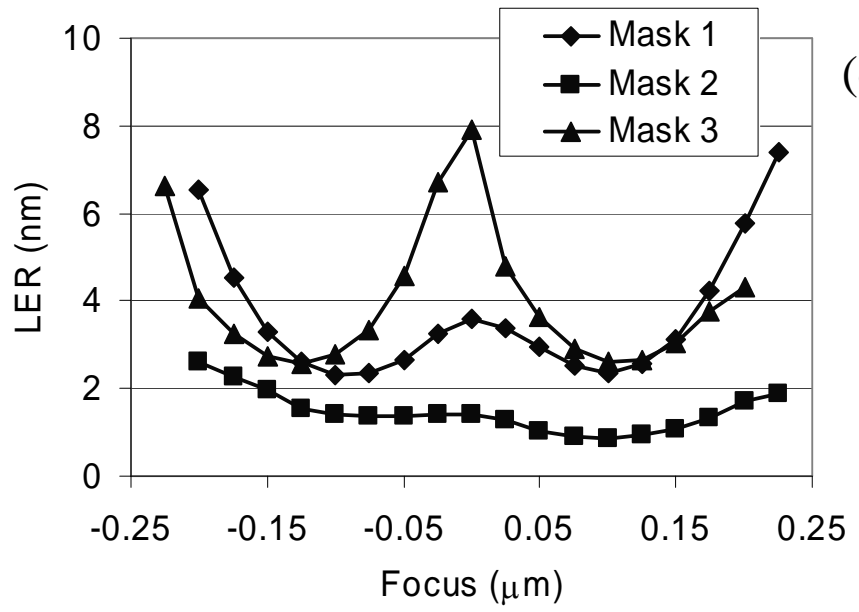

(c) 
Naulleau, et al., Fig. 7

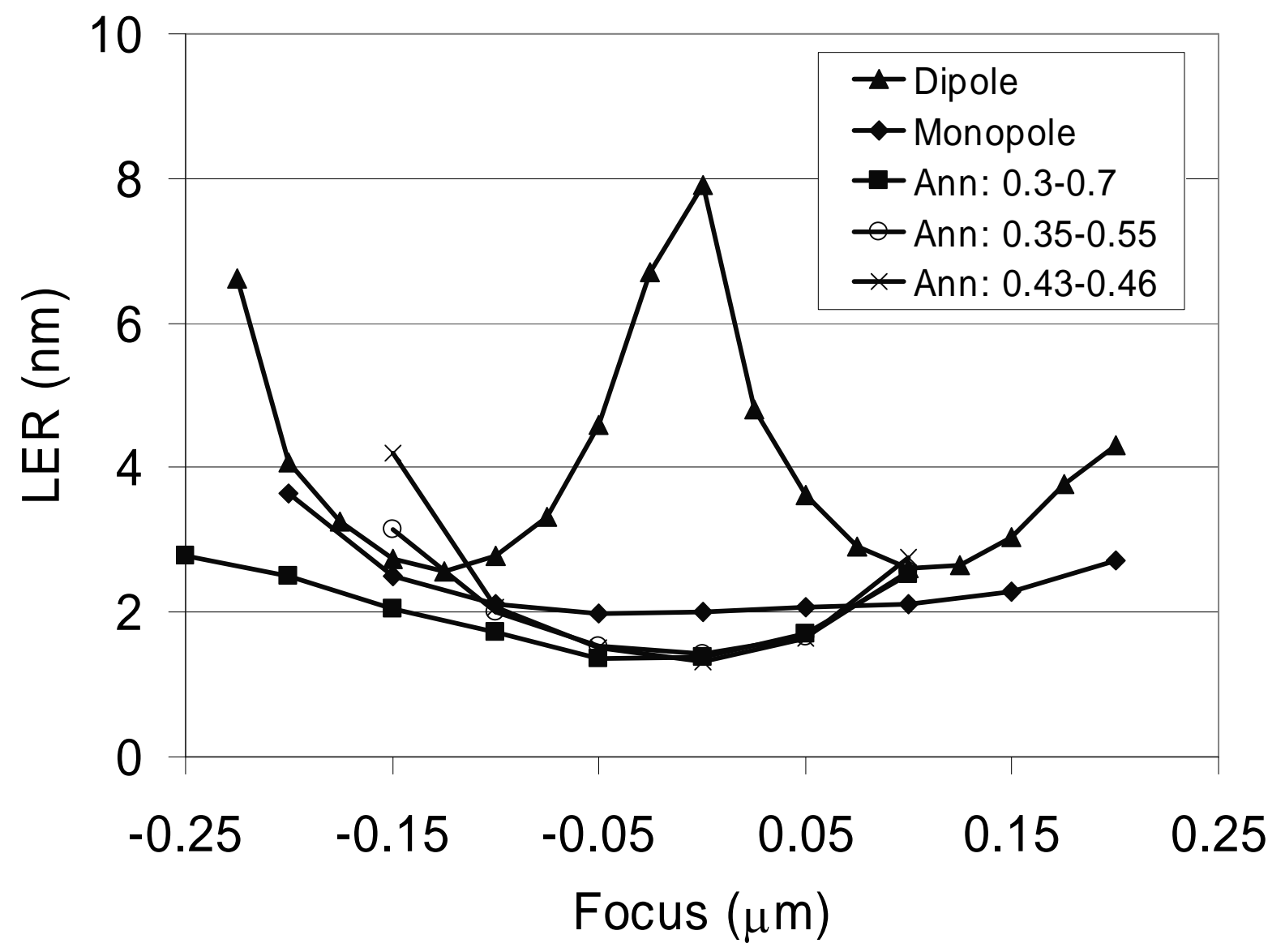


Naulleau, et al., Fig. 8

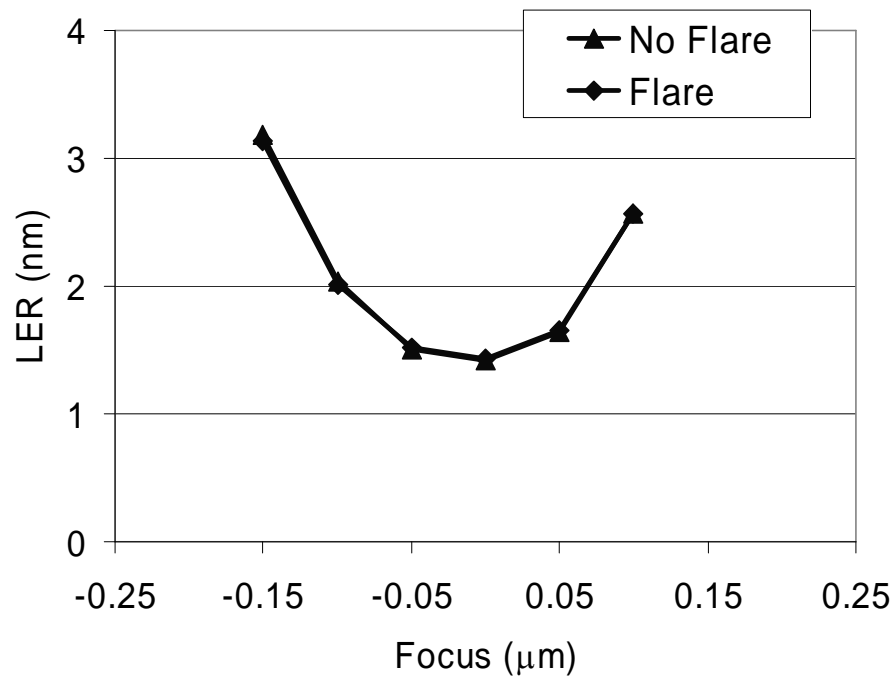

(a)

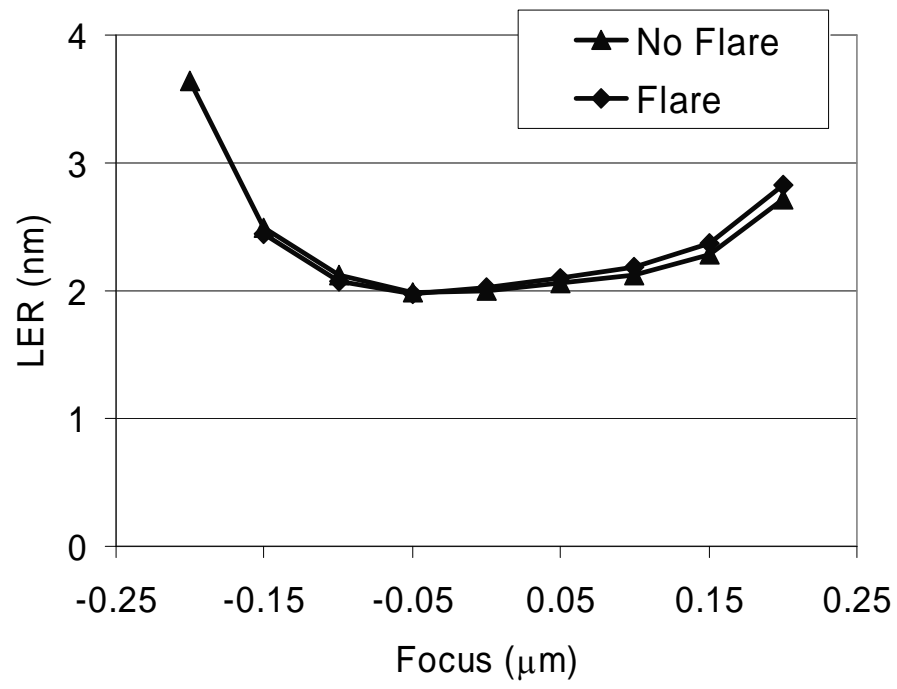

(b)

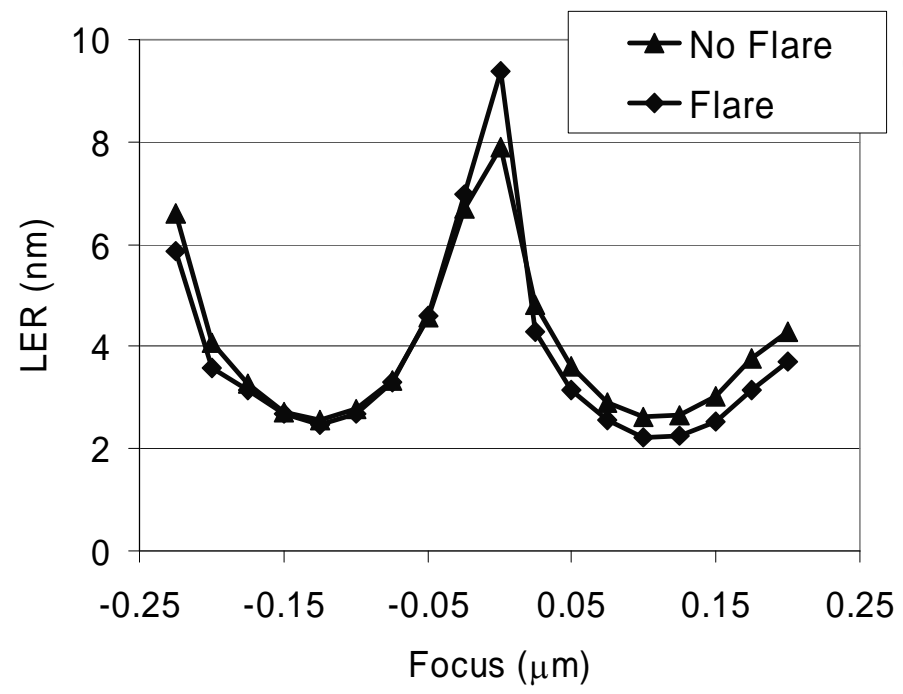

(c) 
Naulleau, et al., Fig. 9

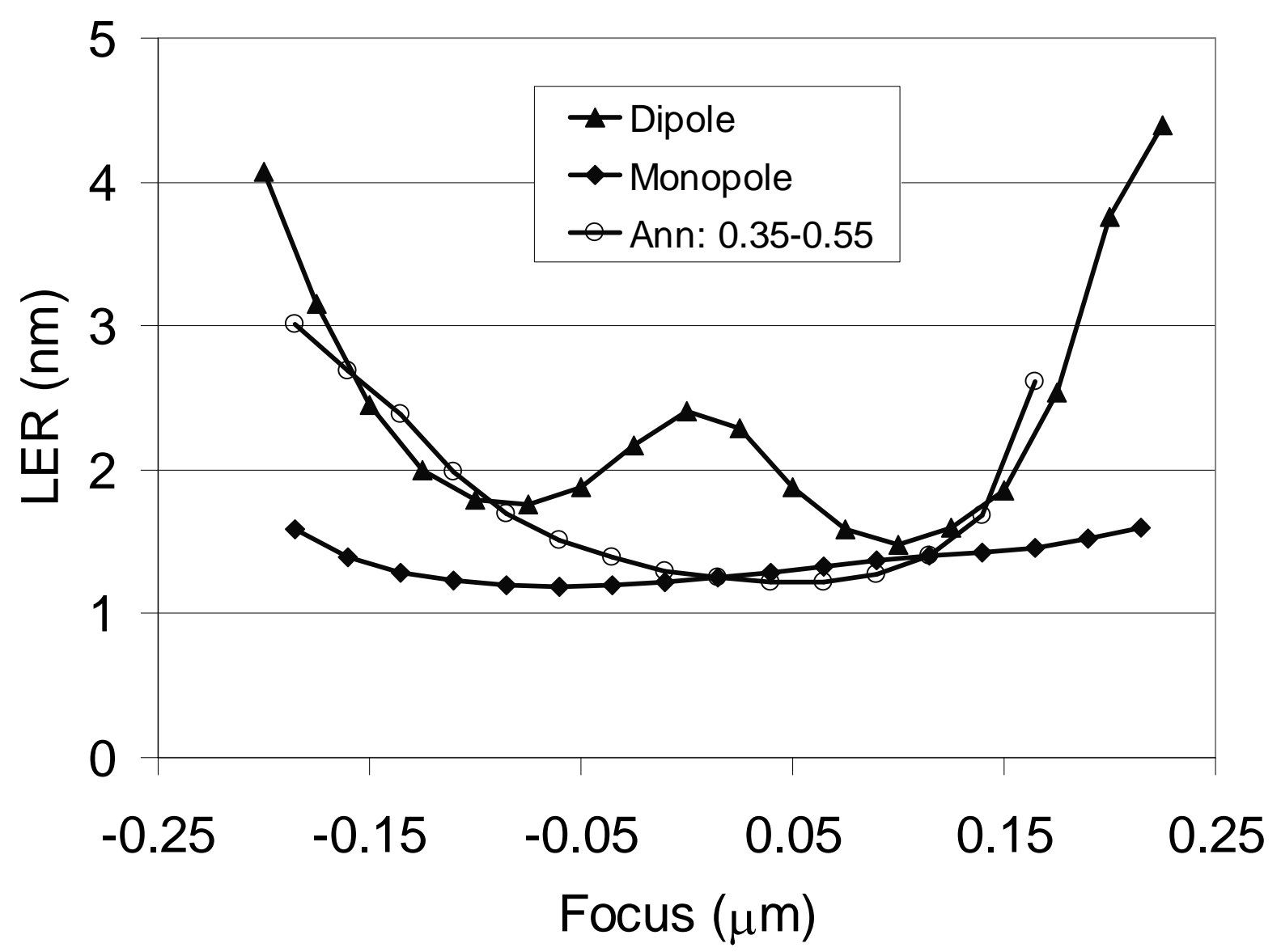


Naulleau, et al., Fig. 10

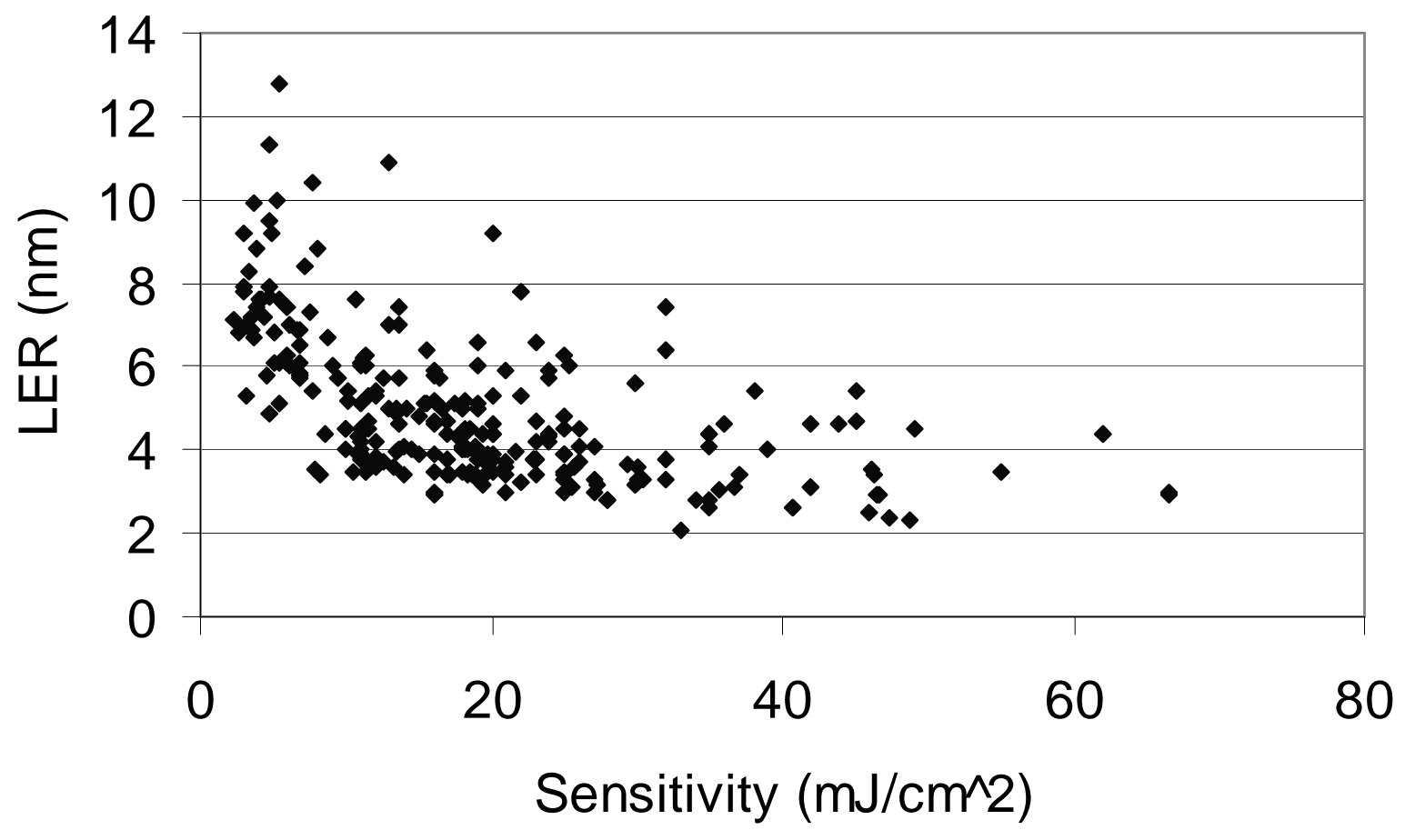



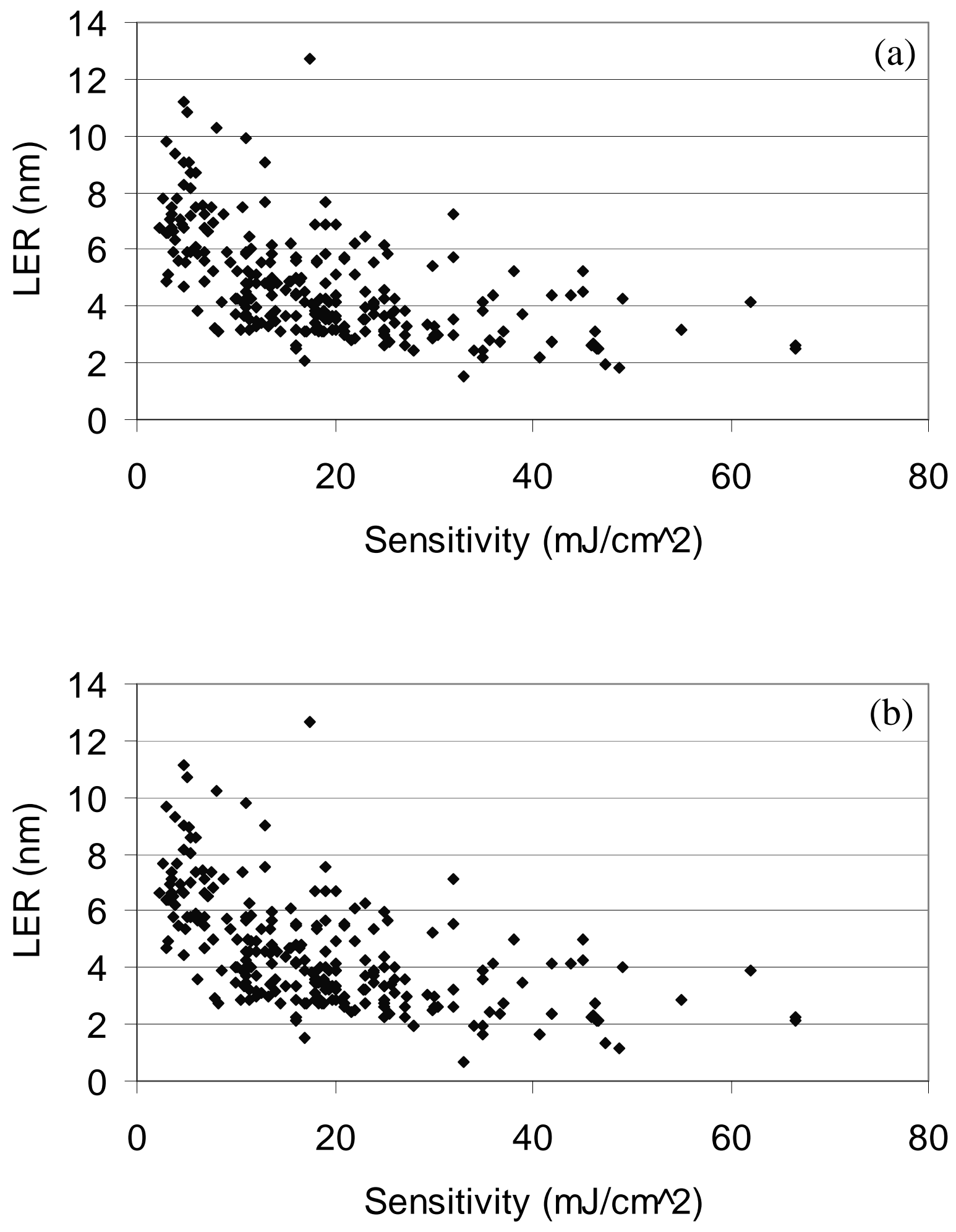